\title{
The Effect of Omega-3 Enriched Oral Nutrition Supplement on Nutritional Indices and Quality of Life in Gastrointestinal Cancer Patients: A Randomized Clinical Trial
}

\author{
Eunbo Sim ${ }^{1}$, Jin-Min Kim², Seung-Min Lee ${ }^{1}$, Moon Jae Chung ${ }^{3}$, Si Young Song ${ }^{3}$, \\ Eun Sun Kim${ }^{4}$, Hoon Jai Chun ${ }^{4}$, Mi-Kyung Sung ${ }^{1 *}$
}

\begin{abstract}
Objective: Gastrointestinal (GI) cancer patients often experience severe malnutrition during cancer therapies due to gastrointestinal dysfunctions including poor digestion and absorption as well as tumor-associated anorexia. In this study, we performed a randomized clinical trial to determine the efficacy of oral nutrition supplement (ONS) enriched with omega-3 fatty acids on nutritional status, quality of life (QOL), and pro-inflammatory indices. Methods: Patients diagnosed with GI cancers were recruited and screened for eligibility. A total of 58 patients were randomly allocated to either the control group $(n=27)$ or the experimental group $(n=31)$. The intervention group received $200 \mathrm{ml}$ ONS twice a day while the control group received routine care. Anthropometrics, Patient-Generated Subjective Global Assessment (PG-SGA) score, QOL score and nutrient intake data were collected at baseline, week 4 and week 8. Blood was drawn for biochemical assessments. Nine patients from each group dropped out of the study Forty patients (18 control patients and 22 intervention patients) completed the study. Results: This study showed that ONS intervention improved PG-SGA scores in the intervention group $(\mathrm{p}<0.01)$. Scores of physical functioning score and role functioning were declined only in the control group and the difference between week 8 and baseline for role functioning was significant $(\mathrm{p}<0.001)$. Fatigue score was steadily decreased in the experiment group, and the differences between week 8 and baseline was significant between two groups $(\mathrm{p}<0.02)$. However, no statistically significant improvement in biochemical markers of nutritional status and pro-inflammatory cytokine concentrations were found. These results suggests that ONS intervention for 8 weeks improves PG-SGA scores and QOL scores in patients undergoing cancer therapy.
\end{abstract}

Keywords: Gastrointestinal cancer- malnutrition- nutritional status- oral nutritional supplement- anti-cancer therapy

Asian Pac J Cancer Prev, 23 (2), 485-494

\section{Introduction}

Non-communicable diseases including cardiovascular diseases (CVD), cancer, chronic respiratory diseases and diabetes have attributed to global premature deaths. The world health organization reported 18.1 million cancer cases in 2018 suggesting 1 in 8 men and 1 in 10 women are likely to develop cancer during their lifetimes (Bray et al., 2018). Most common cancers in men include lung, prostate, colorectum, stomach and liver cancers, while those of in women are breast, colorectum, lung, cervix uteri and thyroid cancers.

Cancer cachexia is a multifactorial condition influencing 50 to $80 \%$ of cancer patients and it is responsible for $20 \%$ of cancer deaths (Warren, 1932; Fearon et al., 2011). Cachexia reveals impairments in energy and protein balance leading weight loss through the loss of skeletal muscle and body fat. However, there has been a lack of consensus to define the severity of cachexia which is necessary in the development of treatment protocol. The cachexia scoring tool, CASCO is a most recent method to calculate cachexia score based on different components including body weight loss, body composition, inflammation, metabolic disturbances, immunosuppression, physical performance, anorexia, and quality of life (QOL) (Argilés et al., 2011). All of these components are associated with cachexia in a very complicated way. A negative energy balance in cachexic patients is attributed by decreased energy intake due to anorexia and inefficient energy use resulted from futile cycle and/or reduced mitochondrial ATP synthesis (Constantinou et al., 2011). Other molecular links between cancer cachexia and the wasting of skeletal muscle and adipose tissue have been described (Argilés et al., 2014). 
Nutritional status of cancer patients affects therapeutic efficacy and the disease survival is influenced by the degree of anorexia. Tumor-associated molecules such as pro-inflammatory cytokines, proteolysis inducing factor and lipid mobilizing factor are known to trigger the loss of appetite. Also, cancer therapy-associated factors such as mucositis, taste aversion, nausea and vomiting induce the loss of appetite. As the efficacy of treatments is determined by the patients' nutritional status, nutrition intervention before and during cancer treatment became a major strategy in cancer care. A recent systemic review showed that $65 \%$ of 28 studies found oral nutrition supplements (ONS) intervention improved one or more markers of nutritional status, immune function and inflammatory responses. Unfortunately, however, there is a lack of consensus on nutrition intervention for cancer patients partly because cancer patients receiving treatments often experience vomiting and dysphagia making dietary intervention very difficult.

Recently, ONS enriched with functional ingredients are available especially to maintain immunological homeostasis and to suppress cancer therapy-induced inflammatory responses (Bozzetti, 2013). Long chain omega-3 fatty acids, eicosapentaenoic acids (EPA) and decosahexaenoic acids (DHA) are most frequently used dietary supplement possibly possessing antiinflammatory activity. Cancer therapy-induced anorexia often accompanies higher circulating concentrations of pro-inflammatory cytokine, which is known to contribute to the metabolic alterations causing the loss of muscle and fat tissue. Therefore, the objective of this study was to evaluate the efficacy of omega-3 fatty acids fortified nutrition supplement intervention on nutritional status, QOL, and pro-inflammatory cytokine concentrations of cancer patients.

\section{Materials and Methods}

\section{Study participants}

Patients were recruited from the Department and Gastroenterology in Korea University Anam Hospital and Yonsei University Severance Hospital between August 2015 and September 2016. Patients ( $\geq 20$ years old) newly diagnosed as having malignant tumor(s) were screened for eligibility and 58 patients who agreed to participate the study were enrolled (27 in the control group and 31 in the intervention group). Patients included in the study were those who are in stages between II to IV receiving one or more cancer therapies without taking any nutritional supplements. Patients who had acute infectious diseases, cardiac insufficiency, hepatic insufficiency or patients receiving hemodialysis were excluded. Dropouts were determined on the basis of clinical needs or participants' request to discontinue. During the course of the study, 18 patients dropped out of the study ( 9 from the control group and 9 from the intervention group) and final analysis was performed in 18 patients in control group and 22 patients in the intervention group. The study was approved by Institutional Review Board of Korea University Anam Hospital (ED14261) and Yonsei University Severance Hospital (4-2015-0436). This study is registered in the
Clinical Research Service, the Korean Centers for Disease Control and Prevention (PRE20210113-004).

\section{Study design and ONS preparation}

Cancer patients eligible for the study were randomly allocated to the experimental group and the control group. The intervention period was 8 weeks long and patients visited study center three times (week 0,4 and 8 ) to have anthropometric, clinical and biochemical measurements taken (Figure 1). Demographic characteristics were collected at baseline. Three-day dietary records were collected at each visit during the intervention. Both control and experimental groups received regular nutrition counseling and education, while only experimental group was asked to take ONS twice a day $(400 \mathrm{ml}, 400$ kcal). Patients were asked to record the amounts of ONS consumed and weekly telephone counseling was used to determine and maintain compliance. Patients in the control group also received weekly telephone call from a trained dietitian for nutrition counseling. ONS was a product of Daesang Wellife (Seoul, South Korea) enriched with omega-3 fatty acids $(70 \mathrm{mg} / 200 \mathrm{ml})$ and arginine $(250 \mathrm{mg} / 200 \mathrm{ml})$. The composition of test product is shown in supplementary Table 1 .

\section{Demographic, anthropometric and clinical measurements}

Study subjects were surveyed for demographic characteristic including sex, birth date, smoking history and drinking habit. Anthropometric measurements were taken at each visit. Body composition was determined using multi-frequency bioelectrical impedance analysis (InBody, Seoul, South Korea). Patient-Generated Subjective Global Assessment (PG-SGA) was used to evaluate improvements in nutritional status of the study participants. A trained dietitian filled out questionnaires in A section of the PG-SGA by face to face interview (weight changes, food intake, clinical symptoms associated with eating behavior, and activities and functions). Body temperature, triceps skinfold thickness, mid-arm muscle circumference were also used to determine PG-SGA score. Dietary intake was assessed by using a 3-day 24hour recall and concurrent dietary records at each visit. A dietitian interviewed patients for their 3-day 24-hour recalls and patients were asked to record their dietary intake by using food model and food weight table. Nutrient intake was analyzed by Can-pro 4.0 (Korean Nutrition Society, Seoul, Korea).

\section{QOL questionnaire}

QOL scores were determined by using EORTC-QLQ C30 (version 3, European Organization for Research and Treatment of Cancer). The questionnaire is composed of 30 questions on three different sections, which are global health status, functional scales and symptom scales.

\section{Biochemical measurements}

Venous blood samples were collected at each visit. Nutritional biomarkers including hemoglobin, albumin, prealbumin, cholesterol, and transferrin were analyzed at the central laboratory of participating Hospitals. Serum concentrations of tumor necrosis factos-alpha (TNF- $\alpha$ ), 
interleukin-6 (IL-6) and interleukin-8 (IL-8) were measured using high-sensitive ELISA detection kit (R\&D, Minneapolis, MN, USA).

\section{Statistical analyses}

Per protocol analyses were applied. All measurements were expressed as mean \pm standard deviation and SPSS program (version 23, 2016, IBM Institute Inc., USA) was used. Baseline anthropometric measures were analyzed using student's t-test if the test statistic follows a normal distribution otherwise Mann-whitney $U$ test was used to compare the control and the intervention groups. Efficacy data were compared using the repeated measured ANOVA test if the test statistic follows a normal distribution. For those data showing skewed distribution, Mann-whitney $U$ test was used to determine the group effect, and Kruskal-wallis test was used to determine time effect and the interaction between group and time. Comparisons between the mean of two different weeks were conducted by paired t-test or Wilcoxon signed rank test. Comparisons between the mean of two groups were conducted by student's t-test of Mann-whitney U test. P-values were determined after Bonferroni corrections for multiple comparisons.

\section{Results}

\section{Demographic and clinical characteristics}

This study included 18 control and 22 ONS intervention participants. Anthropometric and cancer characteristics measures were not significantly different between two groups (Table 1). The average body weight loss was $5.06 \%$ in the control group and $6.01 \%$ in the intervention group. Primary diagnosis sites in the control group were 1 esophagus, 1 duodenum, 5 stomach, 4 pancreas, 3 colon, 1 cecum, 3 rectum and those of the ONS group were 1 esophagus, 3 gallbladder, 1 duodenum, 3 stomach, 8 colon. Percentage of patients in different cancer stage
Oral Nutritional Supplement Improves QOL in Cancer Patients

were $11.1 \%$ (stage II), $16.7 \%$ (stage III), $72.2 \%$ (stage IV) in the control group and $9.1 \%$ (stage II), $36.4 \%$ (stage III), $54.5 \%$ (stage IV) in the intervention group. Majority of the patients received chemotherapy or surgery + chemotherapy in both groups. Among 58 patients enrolled, 18 patients dropped out of the study. Reasons for drop-outs during the protocol were the transfer to another hospital $(n=3)$, symptom of nausea $(n=2)$, refusal to continue due to poor conditions $(n=8)$. Five of the patients died during the course of the study.

\section{Dietary intake}

We have also analyzed basal dietary intake using 3-day 24-hour recall and dietary intake record data (Supplementary Table 2). Basal dietary intake did not include nutrient intake supplied by ONS intervention. Results showed no difference within and between groups except vitamin $\mathrm{K}$, which was higher among the control group patients. Comparisons of dietary intake including ONS showed that total calorie (week 4 and 8), carbohydrate (week 8), fat (week 4 and 8) and protein (week 4 and 8) intake were higher in the intervention group compared to those of the control group (Supplementary Table 3). Also vitamin D, vitamin E, vitamin C, vitamin B-6, calcium, phosphorus, magnesium, zinc and selenium intake were higher in the intervention group compared to those of the control group.

\section{$P G-S G A$ scores}

PG-SGA scores at baseline were 11.9 and 12.4 in the control and the intervention group, respectively (Table 2). Repeated measure ANOVA indicated PG-SGA scores were significantly improved depending on study duration in both groups. No significant difference between groups was observed. However, the paired t-test between week 0 and week 8 showed significant improvements only in the intervention group.

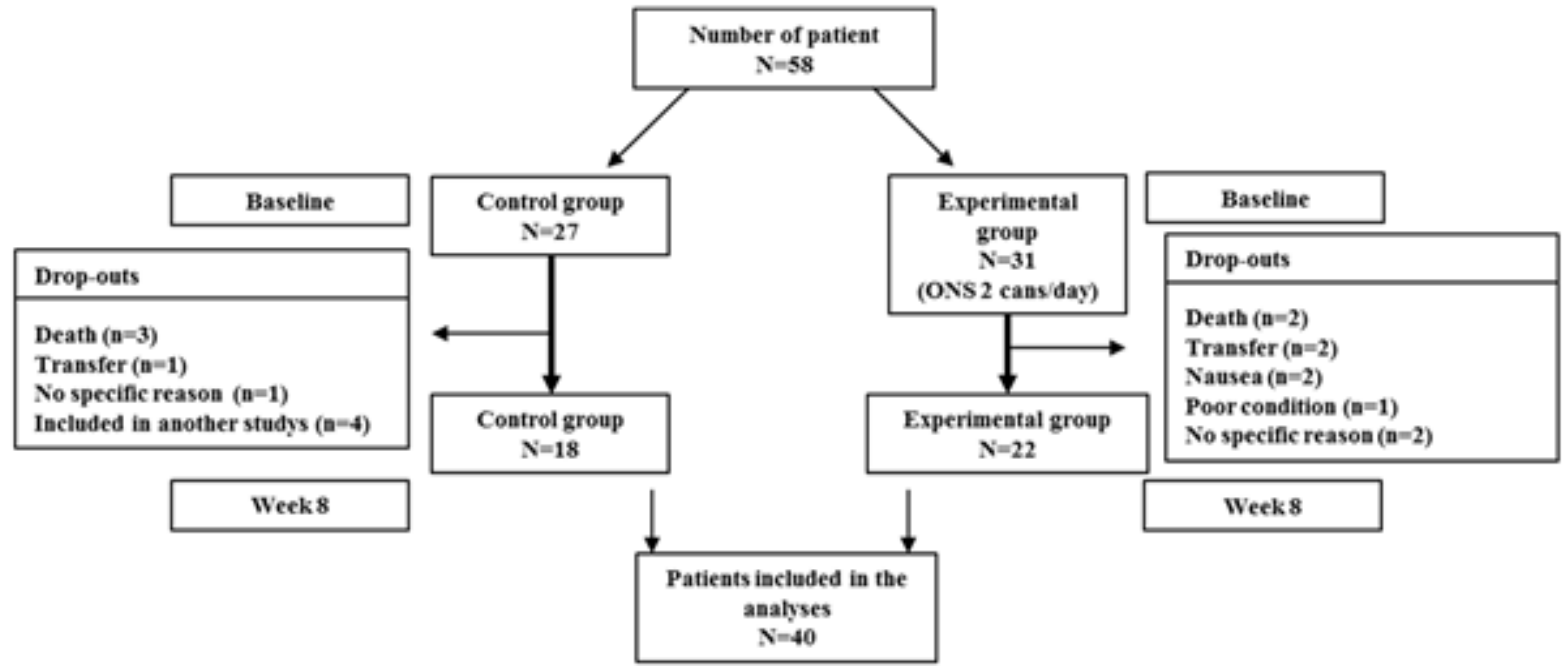

Figure 1. Patient Enrollment and Drop-Out Shown in Consort Flowchart. Patients ( $\geq 20$ years old) newly diagnosed as having malignant tumor(s) were screened for eligibility and 58 patients who agreed to participate the study were enrolled. Dropouts were determined on the basis of clinical needs or participants' request to discontinue. During the course of the study, 18 patients dropped out of the study and final analysis was performed in 18 patients in control group and 22 patients in the intervention group. 
Table 1. Baseline Characteristics of the Intervention and Control Group Patients

\begin{tabular}{|c|c|c|c|}
\hline & Control $(\mathrm{n}=18)$ & Experiment $(\mathrm{n}=22)$ & p-value \\
\hline Age (years) & $65.39 \pm 2.44^{\mathrm{a}}$ & $63.64 \pm 1.79$ & $0.558^{\S}$ \\
\hline Height (cm) & $160.87 \pm 2.29$ & $164.22 \pm 1.19$ & $0.206^{\S}$ \\
\hline Body weight before diagnosis (kg) & $61.86 \pm 2.30$ & $64.88 \pm 1.76$ & $0.296^{\S}$ \\
\hline Body weight changeł (\%) & $-5.06 \pm 0.65$ & $-6.01 \pm 0.99$ & $0.392^{\natural}$ \\
\hline \multicolumn{4}{|l|}{ Sex , no. of subjects $(\%)$} \\
\hline Male & $13(72.2)^{b}$ & $19(86.4)$ & $0.430^{\dagger \dagger}$ \\
\hline Female & $5(27.8)$ & $3(13.6)$ & \\
\hline \multicolumn{4}{|l|}{ Diagnosis site, no. of subjects $(\%)^{\dagger \dagger}$} \\
\hline Esophagus & $1(5.6)$ & $1(4.5)$ & $0.092^{\dagger \dagger}$ \\
\hline Gallbladder & $0(0.0)$ & $3(13.7)$ & \\
\hline Duodenum & $1(5.6)$ & $1(4.6)$ & \\
\hline Pancreas & $5(27.8)$ & $3(13.6)$ & \\
\hline Stomach & $4(22.2)$ & $6(27.3)$ & \\
\hline Colon & $3(16.7)$ & $8(36.4)$ & \\
\hline Cecum & $1(5.6)$ & $0(0.0)$ & \\
\hline Rectum & $3(16.7)$ & $0(0.0)$ & \\
\hline \multicolumn{4}{|l|}{ Cancer stage, no. of subjects $(\%)^{\dagger \dagger}$} \\
\hline Stage II & $2(11.1)$ & $2(9.1)$ & $0.397^{\dagger \dagger}$ \\
\hline Stage III & $3(16.7)$ & $8(36.4)$ & \\
\hline Stage IV & $13(72.2)$ & $12(54.5)$ & \\
\hline \multicolumn{4}{|l|}{ Treatment, no. of subjects $(\%)^{\dagger \dagger}$} \\
\hline Chemotherapy & $10(55.6)$ & $16(72.7)$ & $0.363^{\dagger \dagger}$ \\
\hline Chemo+Radiation & $0(0.0)$ & $1(4.6)$ & \\
\hline Surgery & $1(5.6)$ & $0(0.0)$ & \\
\hline Chemo+Surgery & $6(33.2)$ & $5(22.7)$ & \\
\hline Chemo+Radiation+Surgery & $1(5.6)$ & $0(0.0)$ & \\
\hline \multicolumn{4}{|l|}{ Smoking, no. of subjects (\%) †† } \\
\hline Never & $8(44.4)$ & $8(36.4)$ & $0.545^{\dagger \dagger}$ \\
\hline Former & $8(44.4)$ & $10(45.5)$ & \\
\hline Current & $2(11.1)$ & $4(18.2)$ & \\
\hline \multicolumn{4}{|l|}{ Drinking, no. of subjects $(\%)^{\dagger \dagger}$} \\
\hline Never & $6(33.3)$ & $8(36.4)$ & $0.242^{\dagger \dagger}$ \\
\hline Former & $9(50.0)$ & $13(59.1)$ & \\
\hline Current & $3(16.7)$ & $1(4.5)$ & \\
\hline \multicolumn{4}{|l|}{ Supplement, no. of subjects $(\%)^{\dagger \dagger}$} \\
\hline Yes & $0(0.0)$ & $3(13.6)$ & $0.238^{\dagger \dagger}$ \\
\hline No & $18(100.0)$ & $19(86.4)$ & \\
\hline ECOG (Pateient performance status) & $0.89 \pm 0.14$ & $1.00 \pm 0.17$ & $0.760^{\star}$ \\
\hline
\end{tabular}

a, Data for age, body weight and sex are mean $\pm \mathrm{SD}(\operatorname{control} \mathrm{n}=18$, experimental $\mathrm{n}=22){ }^{\mathrm{b}}$, Data for diagnostic site, cancer stage, treatment, smoking, drinking, supplement, and ECOG are numbers (\%) of subjects; $\$$, p-value for differences between two groups were calculated by Student's t-test; ", p-value for differences between two groups were calculated by Mann-Whitney U test; ${ }^{\dagger \dagger} \mathrm{p}$-value for differences between two groups were calculated by Fisher test; Abbreviation: EGOC-Eastern Cooperative Oncology Group

QOL scores

QOL scores at baseline, week 4 and week 8 are presented in Table 3. The global health status scores were increased only in the intervention group. Repeated measure ANOVA did not show significant group effects on global health status score, however the paired t-test showed a significant increase at week 8 compared to baseline score. Among functional scales, role function score was significantly decreased in the control group at week 8 , while that of the intervention group did not change. There was a group effect at $p=0.056$. Differences in role functioning (baseline vs. week 8) were significant between the control and intervention group. Also, the paired t-test between weeks showed significant decreases in role functioning scores only in the control group. Fatigue symptom scores were steadily increased in the 
Table 2. The Changes of PG-SGA Scores During the Intervention Period

\begin{tabular}{|c|c|c|c|c|c|c|c|}
\hline & \multirow{3}{*}{ Week 0} & \multirow{3}{*}{ Week 4} & \multirow{3}{*}{ Week 8} & \multirow{2}{*}{\multicolumn{2}{|c|}{$\begin{array}{c}\text { Repeated measure } \\
\text { ANOVA }^{\S}\end{array}$}} & \multirow{3}{*}{$\begin{array}{c}\text { Paired t-test }{ }^{\pi} \\
\text { p-value } \\
\text { Week } 0 \text { vs Week } 8\end{array}$} & \multirow{3}{*}{$\begin{array}{c}\text { Student's t-test } \\
\text { p-value } \\
\text { Week8 }\end{array}$} \\
\hline & & & & & & & \\
\hline & & & & Source & p-value & & \\
\hline${\mathrm{PG}-\mathrm{SGA}^{\dagger}}^{\dagger}$ & & & & Time & 0.002 & & \\
\hline Control & $11.89 \pm 0.69$ & $11.78 \pm 0.90$ & $10.67 \pm 0.67$ & Group & 0.137 & 0.190 & 0.118 \\
\hline Experiment & $12.36 \pm 0.66$ & $9.50 \pm 0.78$ & $8.95 \pm 0.80$ & Time*Group & 0.087 & 0.001 & \\
\hline
\end{tabular}

a, Data are expressed in means \pm standard deviations; $\$$, Time effect, group effect, and the interaction between time and group were determined; ', Differences in values between week 0 and week 8 within the group were determined; Abbreviation: PG-SGA-Patient-Generated Subjective Global Assessment

Table 3. Differences in QOL Scores Following 4 and 8 Weeks of Intervention in Study Participants under Cancer Therapy

\begin{tabular}{|c|c|c|c|c|c|c|c|}
\hline & \multirow{3}{*}{ Week 0} & \multirow{3}{*}{ Week 4} & \multirow{3}{*}{ Week 8} & \multirow{2}{*}{\multicolumn{2}{|c|}{$\begin{array}{c}\text { Repeated measure } \\
\text { ANOVA }\end{array}$}} & \multirow{3}{*}{$\begin{array}{c}\text { Paired t-test }{ }^{\ddagger} \\
\text { p-value } \\
\text { Week } 0 \text { vs Week } 8\end{array}$} & \multirow{3}{*}{$\begin{array}{c}\text { Student's t-test }{ }^{\dagger \dagger} \\
\text { p-value } \\
\text { Week } 8\end{array}$} \\
\hline & & & & & & & \\
\hline & & & & Source & p-value & & \\
\hline \multicolumn{8}{|c|}{ Global health status } \\
\hline & & & & Time & 0.130 & & \\
\hline Control & $62.50 \pm 5.40^{\mathrm{a}}$ & $65.05 \pm 4.63$ & $65.97 \pm 4.86$ & Group & 0.765 & 0.335 & 0.393 \\
\hline Experiment & $55.11 \pm 6.15$ & $64.96 \pm 5.57$ & $71.59 \pm 4.34$ & Time*Group & 0.363 & 0.001 & \\
\hline \multicolumn{8}{|c|}{ Functional scales } \\
\hline Physical & & & & Time & 0.55 & & \\
\hline Control & $79.63 \pm 3.71$ & $71.67 \pm 6.03$ & $68.15 \pm 7.20$ & Group & 0.056 & 0.077 & 0.064 \\
\hline Experiment & $82.73 \pm 4.29$ & $79.39 \pm 4.02$ & $83.48 \pm 3.23$ & Time*Group & 0.393 & 0.815 & \\
\hline Role & & & & Time & 0.401 & & \\
\hline Control & $84.20 \pm 5.95$ & $64.81 \pm 8.72$ & $65.74 \pm 7.91$ & Group & 0.399 & 0.001 & 0.135 \\
\hline Experiment & $79.32 \pm 4.90$ & $77.27 \pm 6.06$ & $79.55 \pm 5.02$ & Time*Group & 0.39 & 0.971 & \\
\hline Emotional & & & & Time & 0.619 & & \\
\hline Control & $87.96 \pm 4.33$ & $81.94 \pm 5.10$ & $86.11 \pm 4.95$ & Group & 0.483 & 0.771 & 0.859 \\
\hline Experiment & $82.20 \pm 4.26$ & $85.23 \pm 3.51$ & $87.12 \pm 3.18$ & Time*Group & 0.797 & 0.253 & \\
\hline Cognitive & & & & Time & 0.601 & & \\
\hline Control & $97.22 \pm 2.02$ & $91.67 \pm 4.10$ & $91.67 \pm 4.31$ & Group & 0.503 & 0.210 & 0.881 \\
\hline Experiment & $92.42 \pm 3.24$ & $94.70 \pm 2.30$ & $92.42 \pm 2.84$ & Time*Group & 0.793 & 0.998 & \\
\hline Social & & & & Time & 0.437 & & \\
\hline Control & $87.04 \pm 3.94$ & $78.70 \pm 4.63$ & $73.15 \pm 7.15$ & Group & 0.930 & 0.078 & 0.211 \\
\hline Experiment & $77.27 \pm 6.16$ & $73.48 \pm 6.27$ & $84.09 \pm 4.71$ & Time*Group & 0.592 & 0.206 & \\
\hline \multicolumn{8}{|c|}{ Symptom scales } \\
\hline Fatigue & & & & Time & 0.844 & & \\
\hline Control & $18.52 \pm 4.67$ & $21.60 \pm 6.25$ & $35.21 \pm 8.15$ & Group & 0.528 & 0.020 & 0.053 \\
\hline Experiment & $28.28 \pm 6.15$ & $21.21 \pm 5.89$ & $16.66 \pm 4.18$ & Time*Group & 0.612 & 0.076 & \\
\hline \multicolumn{4}{|c|}{ Nausea and vomiting } & Time & 0.397 & & \\
\hline Control & $3.70 \pm 2.15$ & $9.26 \pm 4.09$ & $11.11 \pm 6.03$ & Group & 0.928 & 0.289 & 0.323 \\
\hline Experiment & $18.94 \pm 6.79$ & $7.58 \pm 4.06$ & $4.55 \pm 3.32$ & Time*Group & 0.169 & 0.027 & \\
\hline Pain & & & & Time & 0.412 & & \\
\hline Control & $7.41 \pm 3.62$ & $4.63 \pm 3.25$ & $12.96 \pm 5.96$ & Group & 0.155 & 0.411 & 0.486 \\
\hline Experiment & $19.70 \pm 5.55$ & $14.39 \pm 5.94$ & $8.33 \pm 3.42$ & Time*Group & 0.243 & 0.083 & \\
\hline Dyspnea & & & & Time & 0.976 & & \\
\hline Control & $7.41 \pm 3.36$ & $5.56 \pm 3.01$ & $22.22 \pm 8.94$ & Group & 0.101 & 0.104 & 0.035 \\
\hline Experiment & $6.06 \pm 2.80$ & $7.58 \pm 3.76$ & $1.51 \pm 1.51$ & Time*Group & 0.256 & 0.083 & \\
\hline Insomnia & & & & Time & 0.259 & & \\
\hline Control & $14.82 \pm 7.24$ & $16.67 \pm 8.20$ & $20.37 \pm 8.15$ & Group & 0.61 & 0.636 & 0.282 \\
\hline Experiment & $31.82 \pm 8.05$ & $12.12 \pm 5.17$ & $10.61 \pm 4.59$ & Time*Group & 0.203 & 0.005 & \\
\hline Appetite loss & & & & Time & 0.365 & & \\
\hline Control & $25.93 \pm 8.76$ & $18.52 \pm 6.16$ & $22.22 \pm 8.52$ & Group & 0.963 & 0.742 & 0.313 \\
\hline Experiment & $27.27 \pm 7.48$ & $24.24 \pm 7.65$ & $12.12 \pm 5.61$ & Time*Group & 0.726 & 0.057 & \\
\hline
\end{tabular}


Table 3. Continued

\begin{tabular}{|c|c|c|c|c|c|c|c|}
\hline & \multirow{3}{*}{ Week 0} & \multirow{3}{*}{ Week 4} & \multirow{3}{*}{ Week 8} & \multirow{2}{*}{\multicolumn{2}{|c|}{$\begin{array}{c}\text { Repeated measure } \\
\text { ANOVA }^{\S}\end{array}$}} & \multirow{3}{*}{$\begin{array}{c}\text { Paired t-test } \\
\text { p-value } \\
\text { Week } 0 \text { vs Week } 8\end{array}$} & \multirow{3}{*}{$\begin{array}{c}\text { Student's t-test }{ }^{\dagger \dagger} \\
\text { p-value } \\
\text { Week } 8\end{array}$} \\
\hline & & & & & & & \\
\hline & & & & Source & p-value & & \\
\hline \multicolumn{4}{|l|}{ Constipation } & Time & 0.567 & & \multirow{3}{*}{0.087} \\
\hline Control & $20.37 \pm 7.69$ & $12.96 \pm 5.48$ & $25.93 \pm 9.55$ & Group & 0.252 & 0.564 & \\
\hline Experiment & $19.70 \pm 7.16$ & $12.12 \pm 5.61$ & $7.58 \pm 3.76$ & Time*Group & 0.653 & 0.029 & \\
\hline \multicolumn{4}{|l|}{ Diarrhea } & Time & 0.298 & & \multirow{3}{*}{0.399} \\
\hline Control & $14.81 \pm 6.72$ & $7.41 \pm 4.31$ & $7.41 \pm 5.08$ & Group & 0.523 & 0.387 & \\
\hline Experiment & $10.61 \pm 5.54$ & $6.06 \pm 2.81$ & $3.03 \pm 2.09$ & Time*Group & 0.671 & 0.233 & \\
\hline \multicolumn{4}{|c|}{ Financial difficulties } & Time & 0.588 & & \multirow{3}{*}{0.046} \\
\hline Control & $20.37 \pm 5.48$ & $27.78 \pm 5.56$ & $27.78 \pm 7.74$ & Group & 0.005 & 0.387 & \\
\hline Experiment & $18.18 \pm 5.69$ & $15.15 \pm 4.77$ & $9.09 \pm 4.49$ & Time*Group & 0.079 & 0.056 & \\
\hline
\end{tabular}

a, Data are expressed in means \pm standard deviations; $\$$, Time effect, group effect, and the interaction between time and group were determined; , Differences in values between week 0 and week 8 within the group were determined; ${ }^{\dagger \dagger}$, Differences in values between two groups at week 8 were determined; All p-values are adjusted for multiple comparison using Bonferroni

control group, while those of the intervention group were decreased. Although repeated measured ANOVA did not find group effects, differences in fatigue score (baseline vs. week 8) were significant between the control and intervention group. Also, the paired t-test between weeks showed that the score at week 8 was significantly higher compared to that of the baseline only in the intervention group. There was a significant time and group interaction $(p=0.027)$ in nausea and vomiting scores showing a steady increase in the control group and a steady decrease in the intervention group. The same trend was observed for insomnia and constipation scores. The paired t-test showed significant worsening of social functioning (baseline vs. week 4) and constipation (baseline vs. week 8). In the meantime, significant improvements were observed for nausea and vomiting (week 4 vs. week 8), dyspnea (week 4 vs. week 8), insomnia (baseline vs. week 4 and baseline vs. week 8) and constipation (baseline vs. week 8).

\section{Biochemical markers}

Biomarkers of nutritional status including albumin, prealbumin, cholesterol, and blood cell counts and sizes were determined (Table 4). Hemoglobin concentration was slightly decreased while transferrin was increased in a time dependent manner, while no significant difference between groups was observed. Pre-albumin was increased in the

Table 4. Differences in Blood Concentrations of Nutritional Markers Following 4 and 8 Weeks of Intervention in Study Participants Under Cancer Therapy

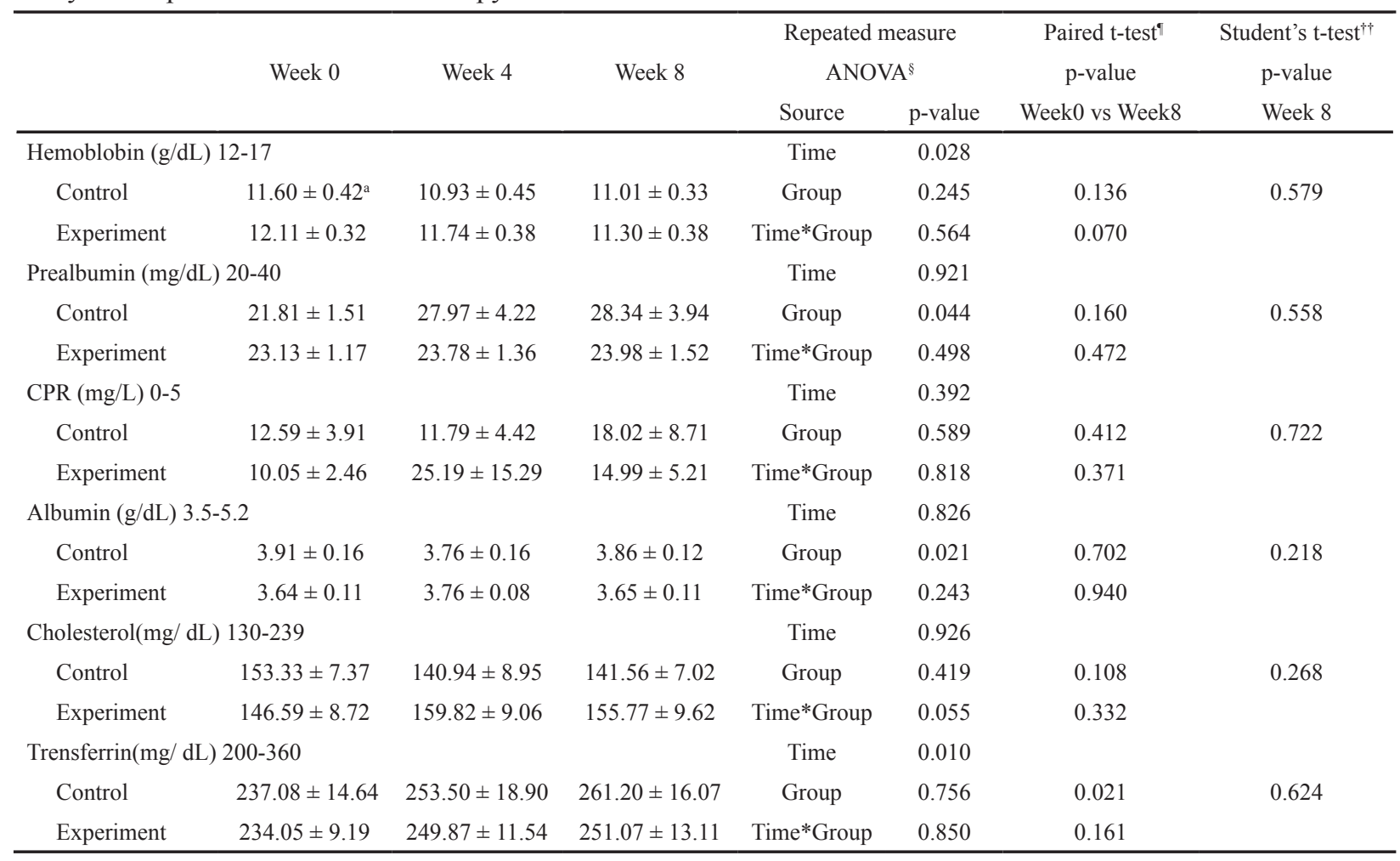

a , Data are expressed in means \pm standard deviations; §, Time effect, group effect, and the interaction between time and group were determined; ' Differences in values between week 0 and week 8 within the group were determined; ${ }^{\dagger}$, Differences in values between two groups at week 8 were determined; All p-values are adjusted for multiple comparison using Bonferroni 
DOI:10.31557/APJCP.2022.23.2.485

Oral Nutritional Supplement Improves QOL in Cancer Patients

Table 5. Blood Concentrations of Inflammatory Cytokines

\begin{tabular}{|c|c|c|c|c|c|c|c|}
\hline & \multirow[t]{2}{*}{ Week 0} & \multirow[t]{2}{*}{ Week 4} & \multirow[t]{2}{*}{ Week 8} & \multicolumn{2}{|c|}{$\begin{array}{c}\text { Repeated measure } \\
\text { ANOVA }\end{array}$} & \multirow{2}{*}{$\begin{array}{c}\text { Paired t-test } \\
\text { p-value } \\
\text { Week } 0 \text { vs Week } 8\end{array}$} & \multirow{2}{*}{$\begin{array}{c}\text { Student's t-test }{ }^{\dagger \dagger} \\
\text { p-value } \\
\text { Week } 8\end{array}$} \\
\hline & & & & Source & $\mathrm{p}$-value & & \\
\hline $\mathrm{Il}-6(\mathrm{pg} / \mathrm{ml})$ & & & & Time & 0.752 & & \\
\hline Control & $7.14 \pm 1.99^{\mathrm{a}}$ & $10.41 \pm 4.21$ & $16.11 \pm 6.60$ & Group & 0.380 & 0.117 & 0.301 \\
\hline Experiment & $6.53 \pm 0.98$ & $13.09 \pm 4.68$ & $9.26 \pm 2.42$ & Time* group & 0.896 & 0.417 & \\
\hline $\mathrm{Il}-8(\mathrm{pg} / \mathrm{ml})$ & & & & Time & 0.999 & & \\
\hline Control & $77.09 \pm 17.54$ & $73.70 \pm 21.29$ & $96.21 \pm 31.68$ & Group & 0.531 & 0.556 & 0.638 \\
\hline Experiment & $65.89 \pm 18.34$ & $81.39 \pm 30.05$ & $77.38 \pm 24.86$ & Time* group & 0.955 & 0.749 & \\
\hline $\mathrm{TNF}-\alpha(\mathrm{pg} / \mathrm{ml})$ & & & & Time & 0.423 & & \\
\hline Control & $18.70 \pm 3.15$ & $18.26 \pm 2.95$ & $20.29 \pm 2.99$ & Group & 0.642 & 0.355 & 0.741 \\
\hline Experiment & $16.64 \pm 1.63$ & $23.79 \pm 6.69$ & $21.67 \pm 2.84$ & Time* group & 0.839 & 0.157 & \\
\hline
\end{tabular}

a, Data are expressed in means \pm standard deviations; $\$$, Time effect, group effect, and the interaction between time and group were determined; ", Differences in values between week 0 and week 8 within the group were determined; ${ }^{\dagger \dagger}$, Differences in values between two groups at week 8 were determined; Abbreviations: IL-6-interleukin-6, IL-8-interleukin-8, TNF- $\alpha$-tumor necrosis factor-alpha

control group with a between group difference $(\mathrm{p}=0.044)$ while albumin concentration was decreased in the control group with a between group difference $(\mathrm{p}=0.021)$. There was a significant interaction between time and group in the concentration of cholesterol showing decreases in the control group and increases in the intervention group. Concentrations of Inflammatory cytokines did not exhibit any differences (Table 5).

\section{Discussion}

In this study, we performed a randomized clinical trial to determine the efficacy of an 8-week intervention of oral nutrition supplement enriched with omega-3 fatty acids on nutritional status, QOL and pro-inflammatory indices in stage II to IV gastrointestinal cancer patients under treatment. Cancer patients under cancer therapy experience loss of appetite, significant weight losses, and low QOL scores. Loss of appetite is induced by therapy-derived gastrointestinal symptoms such as nausea, vomiting and mucositis. Cancer cachexia also attributes to significant weight losses in patients in association with elevated inflammatory responses. To lessen symptoms of cancer cachexia, proper nutrition intervention using ONS containing possible anti-inflammatory compounds has been suggested.

Severe weight loss and malnutrition in cancer cachexia often leads to discontinuation of cancer treatment followed by decreased cancer survival. Therefore, nutrition screening and early nutrition intervention may prevent severe malnutrition (Schmidt et al., 2013; Um et al., 2014) and associated cancer deaths. Despite the malnutrition originated from cancer cachexia differs from other diseaseoriented or age-related malnutrition, nutrition screening tools specifically designed for cancer patients are sparse. PG-SGA is the most widely accepted tools for assessing nutritional status of cancer patients (Mendes et al., 2019). PG-SGA has been suggested as a tool reflecting dynamic nutritional status of the patient compared to other nutrition assessment tools because it scores acute weight changes in addition to intermediate or chronic weight change (Jager-Wittenaar and Ottery, 2017). Although we found no significant improvement in PG-SGA scores between two groups in the repeated measure analyses possibly due to the small sample size and wide variability in the degree of weight loss in study subjects, within-group comparisons showed a significantly decrease in PG-SGA score only in the nutrition intervention group. Despite PG-SGA score well represents the nutritional status of cancer patients, ONS intervention studies have shown mixed results in terms of their efficacies to improve PG-SGA score of patients undergoing cancer therapies. In a recent study, stage III and IV esophageal cancer patients undergoing radiotherapy were subjected to either ONS intervention or routine care during in hospital treatment period (Yang et al., 2020). Study results showed significantly improved indices of nutritional status including PG-SGA scores in the intervention group. Another study in pancreatic and bile duct cancer patients showed that 8 weeks of nutrition intervention significantly improved PG-SGA score (Kim et al., 2019). Although \% weight losses at baseline were not mentioned, $66.7 \%$ and $57.9 \%$ of the study subjects in the intervention and control group were categorized in stage IV implying severe weight loss. In our study, we included subjects whose weight loss were between 5 to $10 \%$ to secure compliance to ONS intervention, which may explain a marginal effect on PG-SGA. In a group of malnourished stomach cancer patients, treatment induced adverse events were associated with body mass index and hypoalbuminemia, but not with PG-SGA where the mean score was 7.42 (Seo et al., 2016) suggesting PG-SGA score responds well to nutrition intervention only in patients with critical need for nutrition intervention (PGA score $\geq 9$ ). In this study, PG-SGA scores were 11.9 in the control group and 12.4 in the intervention group at baseline, and the score was improved and stabilized in the intervention group while that of the control group showed little difference.

Blood biomarkers assessing nutritional status are also used in this study. We previously reported a systematic review on the efficacy of ONS intervention to improve nutritional status of cancer patients (Kim and Sung, 2016). Among 28 qualified intervention studies published between 2001 and 2015, 20 studies used blood biomarkers 
mostly albumin, prealbumin and transferrin as biomarkers of nutritional status. Only 3 among 20 studies found significant improvement in selected biomarkers in the intervention group. In post-discharge colorectal cancer surgery patients who were in ONS intervention group showed reduced skeletal muscle loss compared to those in the control group, however, no difference was found in other biochemical markers (Tan et al., 2021). Similar results were observed when the patients were treated with home enteral nutrition (Li et al., 2019). In this study, we also found no significant difference in nutritional status markers between two groups. Albumin and prealbumin are biomarkers most frequently used to monitor nutritional status of patients who are at risk of malnutrition. However, blood albumin concentration has been known to be associated with other physiological factors including age, circulating estrogen concentration, chronic blood loss and iron deficiency (Banh, 2006) suggesting albumin alone might not be an accurate marker for nutritional intervention in cancer patients (Friedman and Fadem, 2010) receiving different treatments. One of the most recent reviews states that serum albumin levels for nutritional monitoring of critically ill patients are influenced by the acute phase response such as inflammation and may not accurately reflect nutritional status (Kubota et al., 2020). Other recent studies combined albumin concentration with other nutritional and inflammatory biomarkers to assess the efficacy of ONS intervention (Hao et al., 2020; Jeon et al., 2020) with better predictions for cancer prognosis. A meta-analysis for nutritional status markers in older adults with cancer indicated that prognostic nutritional index based on albumin concentration + lymphocyte counts was associated with overall survival (Bullock et al., 2020). Another study showed that nasopharyngeal cancer patients receiving chemoradiotherapy + nutrition intervention had higher white blood cell counts and platelet counts when compared to those receiving chemoradiotherapy + routine care (Yang et al., 2020).

Based on the fact that chronic inflammatory conditions attributes to cancer cachexia leading body weight loss especially muscle wasting, ONS used in this study was fortified with omega-3 fatty acids possessing anti-inflammatory functions. In fact, recent studies on nutritional intervention in cancer patients focus more to provide ingredients having anti-inflammatory function in combination with muscle building amino acids. Hyper and chronic inflammatory conditions in cancer patients are created not only by cancer therapies but also metabolic alterations associated with tumor growth (Biswas and Acharyya, 2020). Chemotherapy and radiotherapy induce inflammatory responses in tissue epithelium especially in digestive tract contributing to mucositis resulting in decreased food intake, malabsorption, diarrhea and severe weight loss. In our previous ONS intervention study, cereal-based ONS products containing chiaseed powder $(5 \% \mathrm{w} / \mathrm{w})$ rich in omega-3 fatty acids lowered pro-inflammatory cytokine production in LPS-stimulated PBMC isolated from cancer patients (Kim and Sung, 2016). ONS enriched with L-arginine, omega-3 fatty acids and ribonucleic acids improved long-term survival of head and neck cancer patients (Boisselier et al., 2020).
Another study showed improved chemotherapy tolerance and prognosis when GI cancer patients were subjected to fish oil-enriched ONS supplementation for 6 months during chemotherapy (Shirai et al., 2017). However, a meta- analysis on effects of enteral nutrition enriched with omega-3 fatty acids in comparison with enteral nutrition without omega-3 fatty acids suggested no significantly different efficacy for nutritional status indices including body weight, body mass index, albumin, wound infection or pneumonia in GI cancer patients during postoperative convalescence (Wan et al., 2020) indicating further clinical trials carefully designed to provide explanations for the association between nutritional status, inflammatory responses and intervention using anti-inflammatory ONS.

However, ONS supplementation for 8 weeks exerted improvements in many of QOL indices. Health-related quality of life (HRQOL) is one of the most important supportive care issue in cancer patients, and a systematic review and meta-analysis to examine the efficacy of ONS intervention in malnourished cancer patients indicated that ONS intervention exerted significant improvements in QOL scores without significant efficacies in anthropometric or biochemical markers (Zhang et al., 2018). We observed improvements in role function score, fatigue symptom score, nausea and vomiting scores, insomnia and constipations scores. A study in bile duct/ pancreatic patients showed ONS intervention for 8 weeks improved fatigue symptoms and increased fat mass (Kim et al., 2019). A meta- analysis to evaluate the efficacy of home enteral nutrition (HERN) in the QOL of discharged gastrointestinal patients also suggested improvements in patient fatigue levels in HERN group compared to that of the control group (Xueting et al., 2020). Eightly $\%$ of cancer patients receiving chemotherapy and/or radiotherapy reported to have fatigue symptoms (Henry et al., 2008). Cancer-related fatigue (CRF) is defined as an unusual, persistent, and subjective sense of tiredness that is not proportional to recent activity and interferes with usual functioning (Malik et al., 2001). Although the present study and other previously mentioned intervention studies reported efficacy of nutritional supplementation in reducing $\mathrm{CRF}$, many others reported nutrition therapy could not prove definite effects on CFR and QOL in cancer patients implying ONS intervention studies tailored for cancer specificity and bioactive ONS ingredients are required (Baguley et al., 2019).

We also found that ONS intervention alleviated symptoms of gastrointestinal symptoms including nausea, vomiting and constipation. Chemotherapeutic agents are known to trigger the secretion of neurotransmitters such as serotonin and substance P mitigating vagal signaling (Adel, 2017) associated with nausea and vomiting. Despite many studies reported a positive association between nutritional supplements and QOL, few studies examine the efficacy of nutritional supplementation on therapy-associated GI symptoms. Nutrition intervention in palliative cancer patients improved patient-rated symptom of nausea and vomiting without differences in other measures (Uster et al., 2018). Home delivery meal service found significantly improved food intake leading a higher QOL (Leedo et al., 2017). Contrary to these findings, 
ONS intervention in patients with nasopharyngeal cancer during chemotherapy improved body weight, BMI and prealbumin, while no difference was observed for QOL Botanical components such as ginger (Saneei Totmaj et al., 2019) and several dietetic interventions, for example the use of dried foods such as toasts and crackers, small and frequent meals have been suggested to relive nausea symptoms (Marx et al., 2016). These results suggests nutritional supplements can improve some of the QOL components which needs evidence-based explanation in mechanistic aspects. Several limitations of this study complicate interpretation. First, the large number of dropouts weakens the strength of the study findings. The attrition rate of the study was $31 \%$ mostly due to the illness of the study participants and ethical considerations in maintain fragile patients. Unfortunately, the absence of follow-up data in the dropouts precluded intention-totreat analyses to strengthen the interpretation of the data. Second, the study participants are diagnosed with different types of gastrointestinal cancer which might contributed to different responses to nutrition intervention with large variations in various markers despite a high compliance for those who completed the study and the selection criteria based on weight loss at diagnosis..

\section{Author Contribution Statement}

Eunbo Sim, Jin-Min Kim, Moon Jae Chung, Eun Sun Kim collected and curated data. Eunbo Sim and MiKyung Sung wrote the draft of the manuscript. Si Young Song, Eun Sun Kim, Hoon Jai Chun and Mi-Kyung Sung conceptualize the study and methodology. Seung-Min Lee and Mi-Kyung Sung supervised the study and finalized the manuscript. Si Young Song, Hoon Jai Chun and Mi-Kyung Sung contributed to funding acquisition. Statements are described at the end of the manuscript.

\section{Acknowledgements}

This study was supported by the Medical Research Center Program (No. 2011-0030074) through National Research Foundation (NRF) grant funded by the Korean government (MSIP). We would like to thank to Ji-In Yoon (Sookmyung Women's University, Seoul, Republic of Korea) for providing technical assistance in manuscript preparation. This work is a part of the Master's degree thesis for Eunbo Sim at Yonsei University.

\section{Conflicts of interest}

There is no conflicts of interest

\section{References}

Adel N (2017). Overview of chemotherapy-induced nausea and vomiting and evidence-based therapies. Am J Manag Care, 23, S259-s65.

Argilés JM, Busquets S, Stemmler B, et al (2014). Cancer cachexia: understanding the molecular basis. Nat Rev Cancer, 14, 754-62.

Argilés JM, López-Soriano FJ, Toledo M, et al (2011). The cachexia score (CASCO): a new tool for staging cachectic
Oral Nutritional Supplement Improves QOL in Cancer Patients cancer patients. J Cachexia Sarcopenia Muscle, 2, 87-93.

Baguley BJ, Skinner TL, Wright ORL (2019). Nutrition therapy for the management of cancer-related fatigue and quality of life: a systematic review and meta-analysis. Br J Nutr, 122, 527-41.

Banh L (2006). Serum proteins as markers of nutrition: What are we treating?. Practical Gastroenterol, 30, 46-64.

Biswas AK, Acharyya S (2020). Cancer-associated cachexia: A systemic consequence of cancer progression. Ann Rev Cancer Biol, 4, 391-411.

Boisselier P, Kaminsky MC, Thézenas S, et al (2020). A doubleblind phase III trial of immunomodulating nutritional formula during adjuvant chemoradiotherapy in head and neck cancer patients: IMPATOX. Am J Clin Nutr, 112, 1523-31.

Bozzetti F (2013). Nutritional support of the oncology patient. Crit Rev Oncol Hematol, 87, 172-200.

Bray F, Ferlay J, Soerjomataram I, et al (2018). Global cancer statistics 2018: GLOBOCAN estimates of incidence and mortality worldwide for 36 cancers in 185 countries. $C A$ Cancer J Clin, 68, 394-424.

Bullock AF, Greenley SL, McKenzie GAG, et al (2020). Relationship between markers of malnutrition and clinical outcomes in older adults with cancer: systematic review, narrative synthesis and meta-analysis. Eur J Clin Nutr, 74, 1519-35.

Cancer Today-International Agency for Research on Cancer (IARC) [Online]. World Health Organization. Available at: https://gco.iarc.fr/today/data/factsheets/cancers/39-Allcancers-fact-sheet.pdf (Accessed 2020/12).

Constantinou C, Fontes de Oliveira CC, Mintzopoulos D, et al (2011). Nuclear magnetic resonance in conjunction with functional genomics suggests mitochondrial dysfunction in a murine model of cancer cachexia. Int J Mol Med, 27, 15-24.

Fearon K, Strasser F, Anker SD, et al (2011). Definition and classification of cancer cachexia: an international consensus. Lancet Oncol, 12, 489-95.

Friedman AN, Fadem SZ (2010). Reassessment of albumin as a nutritional marker in kidney disease. J Am Soc Nephrol, 21, 223-30.

Hao J, Chen C, Wan F, et al (2020). Prognostic value of pre-treatment prognostic nutritional index in esophageal cancer: A systematic review and meta-analysis. Front Oncol, 10, 797.

Henry DH, Viswanathan HN, Elkin EP, et al (2008). Symptoms and treatment burden associated with cancer treatment: results from a cross-sectional national survey in the U.S. Support Care Cancer, 16, 791-801.

Jager-Wittenaar H, Ottery FD (2017). Assessing nutritional status in cancer: Role of the patient-generated subjective global assessment. Curr Opin Clin Nutr Metab Care, 20, 322-9.

Jeon CH, Park KB, Jung YJ, et al (2020). Modified controlling nutritional status score: A refined prognostic indicator depending on the stage of gastric cancer. Surg Oncol, 34, 261-9.

Kim JM, Sung MK (2016). The efficacy of oral nutritional intervention in malnourished cancer patients: A systemic review. Clin Nutr Res, 5, 219-36.

Kim SH, Lee SM, Jeung HC, et al (2019). The Effect of nutrition intervention with oral nutritional supplements on pancreatic and bile duct cancer patients undergoing chemotherapy. Nutrients, 11, 1145.

Kubota T, Shoda K, Konishi H, et al (2020). Nutrition update in gastric cancer surgery. Ann Gastroenterol Surg, 4, 360-8.

Leedo E, Gade J, Granov S, et al (2017). The Effect of a home delivery meal service of energy- and protein-rich meals on quality of life in malnourished outpatients suffering from 
lung cancer: A randomized controlled trial. Nutr Cancer, 69, 444-53.

Li X, Zhou J, Chu C, et al (2019). Home enteral nutrition may prevent myelosuppression of patients with nasopharyngeal carcinoma treated by concurrent chemoradiotherapy. Head Neck, 41, 3525-34.

Malik UR, Makower DF, Wadler S (2001). Interferon-mediated fatigue. Cancer, 92, 1664-8.

Marx W, Kiss N, McCarthy AL, et al (2016). Chemotherapyinduced nausea and vomiting: A narrative review to inform dietetics practice. J Acad Nutr Diet, 116, 819-27.

Mendes NP, Barros TA, Rosa COB, et al (2019). Nutritional screening tools used and validated for cancer patients: A systematic review. Nutr Cancer, 71, 898-907.

Saneei Totmaj A, Emamat H, Jarrahi F, et al (2019). The effect of ginger (Zingiber officinale) on chemotherapy-induced nausea and vomiting in breast cancer patients: A systematic literature review of randomized controlled trials. Phytother Res, 33, 1957-65.

Schmidt KN, Olson K, Kubrak C, et al (2013). Validation of the head and neck patient symptom checklist as a nutrition impact symptom assessment tool for head and neck cancer patients. Support Care Cancer, 21, 27-34.

Seo SH, Kim SE, Kang YK, et al (2016). Association of nutritional status-related indices and chemotherapy-induced adverse events in gastric cancer patients. BMC Cancer, 16, 900.

Shirai Y, Okugawa Y, Hishida A, et al (2017). Fish oil-enriched nutrition combined with systemic chemotherapy for gastrointestinal cancer patients with cancer cachexia. Sci Rep, 7, 4826.

Tan S, Meng Q, Jiang Y, et al (2021). Impact of oral nutritional supplements in post-discharge patients at nutritional risk following colorectal cancer surgery: A randomised clinical trial. Clin Nutr, 40, 47-53.

Um MH, Choi MY, Lee SM, et al (2014). Intensive nutritional counseling improves PG-SGA scores and nutritional symptoms during and after radiotherapy in Korean cancer patients. Support Care Cancer, 22, 2997-3005.

Uster A, Ruehlin M, Mey S, et al (2018). Effects of nutrition and physical exercise intervention in palliative cancer patients: A randomized controlled trial. Clin Nutr, 37, 1202-9.

Wan GY, Zheng LY, Li HQ, et al (2020). Effects of enteral nutritional rich in $n-3$ polyunsaturated fatty acids on the nutritional status of gastrointestinal cancer patients: a systematic review and meta-analysis. Eur J Clin Nutr, 74, 220-30.

Warren S (1932). The immediate causes of death in cancer. $A M$ J Med Sci, 184, 610-5.

Xueting H, Li L, Meng Y, et al (2020). Home enteral nutrition and oral nutritional supplements in postoperative patients with upper gastrointestinal malignancy: A systematic review and meta-analysis. Clin Nutr, 40, 3082-93

Yang L, Gao J, Zhou Y, et al (2020). Effect of oral nutritional supplements on patients with esophageal cancer during radiotherapy. Cancer Biother Radiopharm, 2020.

Zhang F, Shen A, Jin Y, et al (2018). The management strategies of cancer-associated anorexia: a critical appraisal of systematic reviews. BMC Complement Altern Med, 18, 236.

This work is licensed under a Creative Commons AttributionNon Commercial 4.0 International License. 